\title{
Profile of tivozanib and its potential for the treatment of advanced renal cell carcinoma
}

\author{
This article was published in the following Dove Press journal: \\ Drug Design, Development and Therapy \\ 20 June 2013 \\ Number of times this article has been viewed
}

\section{Lance Cowey}

Baylor-Sammons Cancer Center, Texas Oncology, PA, US Oncology Research, Dallas, TX, USA
Correspondence: C Lance Cowey Baylor-Sammons Cancer Center, Texas Oncology, PA, US Oncology Research, 34I0 Worth Street, Ste 300, Dallas, TX 75246, USA Email lance.cowey@usoncology.com

\begin{abstract}
Tivozanib is a novel vascular endothelial growth factor receptor tyrosine kinase inhibitor (VEGF TKI). Among other VEGF TKIs, tivozanib stands apart due to its selective kinase inhibitory properties as well as its high potency for inhibiting VEGF receptors 1 and 2. Tivozanib has been evaluated in several clinical trials including a Phase I and Phase II trial demonstrating safety and efficacy for patients with advanced clear cell renal cell carcinoma (RCC). A pivotal randomized Phase III trial comparing the front-line use of tivozanib to sorafenib in patients with metastatic clear cell RCC has been reported. The clinical development of tivozanib and results of these important studies will be reviewed. Also, the potential placement of tivozanib among currently US Food and Drug Administration approved agents for advanced RCC will be discussed.
\end{abstract}

Keywords: tivozanib, AV-951, metastatic renal cell carcinoma, RCC, VEGF inhibitor, targeted therapy, angiogenesis inhibitor

\section{Introduction}

Renal cell carcinoma (RCC) is a common malignancy with approximately 64,000 cases diagnosed each year and 13,500 deaths. ${ }^{1}$ Most cases are localized and can be cured with surgical resection; however, a large portion of patients go on to develop distant recurrence. Additionally, many patients are found to have unresectable disease or distant metastases at the time of initial diagnosis. Patients with unresectable or metastatic disease are collectively termed to have advanced RCC. There are several histologic types of RCC with the most common type being that of clear cell RCC (75\%) and the remainder of subtypes being papillary, chromophobe, and oncocytomas. Also, mixed histologies can occur as well as de-differentiated types including sarcomatoid and xp11.2 translocation types which are unusually aggressive in their clinical behavior.

An increased understanding of RCC molecular biology has led to the development of several targeted therapies which have a high level of activity. A pivotal finding has been the association of von Hippel-Lindau gene (VHL) aberrance with clear cell RCC. VHL disease is a hereditary genetic syndrome which results in loss of the VHL function and clinical characteristics include the potential for development of clear cell RCC tumors, hemangiomas, and visceral cysts. ${ }^{2}$ The germline loss of VHL results in the stabilization of a family of transcription factors known as hypoxia inducible factors (HIF). ${ }^{3}$ These transcription factors when present are able to induce transgenic activation of several target genes, including vascular endothelial growth factor (VEGF), platelet derived growth factor, fibroblast growth factor, and several other genes which can promote tumor cell survival and proliferation. Interestingly, most sporadic RCC 
cases also contain loss of VHL function through a variety of mechanisms which make the cancer susceptible to targeting the end-products of HIF transactivation, such as VEGF. ${ }^{4,5}$

VEGF functions as a growth factor which binds to VEGF receptors on vascular endothelial cells and stromal support cells, resulting in vascular budding, growth, and maintenance. ${ }^{6}$ VEGF is one of many factors which result in the angiogenic phenotype which is a hallmark of cancer. A variety of agents have been evaluated which inhibit components of the VEGF pathway in RCC tumors, resulting in significant clinical activity. One of the newest agents which have been developed that target the VEGF pathway is tivozanib. This review will focus on the clinical development of tivozanib and the potential placement of this novel agent among other approved drugs used to treat this disease.

\section{Overview of currently available therapeutics for advanced RCC}

Advanced RCC has several unique characteristics compared to other cancers. It is a cancer that is largely insensitive to traditional cytotoxic chemotherapies as evidenced by numerous negative Phase II studies which evaluated a wide variety of these drugs. ${ }^{7}$ Additionally, RCC is one of a few cancers that can respond to immunotherapy. The cytokines interferon and interleukin-2 (IL-2) have demonstrated response rates of approximately $10 \%$, with some responses to IL-2 being complete and durable. ${ }^{8}$ While these drugs have garnered US Food and Drug Administration (FDA) approval, most patients do not benefit and the side-effects of these agents are significant. ${ }^{8}$ The Memorial Sloan Kettering Cancer Center (MSKCC) or Motzer criteria is an important risk stratification tool which was developed in the era of immune-based therapies. ${ }^{9}$ These criteria include poor performance status, anemia, hypercalcemia, elevated lactate dehydrogenase, and absence of prior nephrectomy. Patients with zero risk factors are deemed good risk, one to two risk factors are intermediate risk, and those with three or more are poor risk. This risk factor tool has played an important role in stratification of patients for clinical trials testing new drugs for RCC over the last decade.

The end of 2005 saw the first of a series of new targeted agents receive FDA approval for use in advanced RCC based on a series of pivotal Phase III trials (see Table 1 for a summary of these trials). These targeted agents have been part of two main classes: VEGF pathway inhibitors and mammalian target of rapamycin (mTOR) pathway inhibitors. Sorafenib, sunitinib, pazopanib, and axitinib are all orally bioavailable vascular endothelial growth factor receptor tyrosine kinase inhibitors (VEGF TKIs) which have gained FDA approval for advanced RCC. These agents have varied potency on the VEGF receptors (Table 2) as well as a varied profile of non-VEGF receptors that they inhibit. Bevacizumab is a monoclonal antibody which binds and clears all isoforms of VEGF A and has been FDA approved for use in front-line RCC patients in combination with interferon. ${ }^{10-13}$ Currently, the VEGF TKIs sunitinib ${ }^{14,15}$ and pazopanib $^{16}$ are also considered evidence-based options for front-line use in advanced RCC; while, axitinib ${ }^{17}$ has been approved for second-line (after failure of front-line VEGF inhibitor, cytokine, or temsirolimus) and sorafenib ${ }^{18,19}$ (and pazopanib also) has shown a high level of activity after frontline cytokine therapy.

The mTOR pathway also has been an important target for $\mathrm{RCC}$ drug development. The mTOR protein is an upstream effector of HIF biosynthesis. It is also a key regulator of several other pathways involved in tumor cell growth and metabolism. Temsirolimus is an intravenous inhibitor of the mTOR complex which has been approved based on a Phase III trial showing improved survival in advanced RCC patients with poor risk disease. ${ }^{20}$ Everolimus is an orally bioavailable mTOR inhibitor which has received FDA approval for patients who have been treated with at least one VEGF TKI. ${ }^{21,22}$

It is in the setting of these available agents that tivozanib has been developed and stands poised to be the next agent approved. Most of these agents have been evaluated with interferon or a placebo as a comparator; however, the newer agents axitinib and tivozanib have been compared to sorafenib.

\section{Phase I development of tivozanib}

Tivozanib (previously known as AV-951) is an orally bioavailable VEGF TKI which potently inhibits VEGF receptors 1,2, and 3. Tivozanib has shown antitumor activity in RCC xenograft models in addition to several other solid tumor models leading to its evaluation in clinical testing. ${ }^{23}$ Tivozanib was studied in a Phase I clinical study of patients with advanced solid tumor malignancies which were resistant to other therapies. In this trial, 41 patients were enrolled to be treated with varied doses of the drug with endpoints including determining the maximum tolerated dose (MTD), doselimiting toxicities (DLT), safety profile, pharmacokinetic profile, and pharmacodynamic profile.

Key criteria for enrollment included an advanced solid tumor malignancy for which no remaining conventional therapy remained, Eastern Cooperative Oncology Group (ECOG) performance score of 2 or less, adequate organ function (including no ongoing cardiac abnormalities, uncontrolled hypertension, or proteinuria of $>3.5 \mathrm{~g} / 24$ hours), 
Table I Summary of pivotal Phase III trials for targeted therapies in advanced RCC

\begin{tabular}{|c|c|c|c|c|c|c|c|}
\hline Agent & MOA & $\begin{array}{l}\text { FDA } \\
\text { approval }\end{array}$ & $\begin{array}{l}\text { Pivotal trial } \\
\text { comparator }\end{array}$ & $\begin{array}{l}\text { Pivotal trial } \\
\text { population }\end{array}$ & $\begin{array}{l}\text { Primary } \\
\text { endpoint }\end{array}$ & Outcome & References \\
\hline Tivozanib & VEGF TKI & Pending & Sorafenib & $\begin{array}{l}\text { Treatment naïv; } \\
\text { one prior non- } \\
\text { VEGF/non- } \\
\text { mTORi allowed }\end{array}$ & PFS & $\begin{array}{l}\text { PFS II.9 months (tivozanib) vs } \\
9.1 \text { months (sorafenib), } P=0.042 \text {; } \\
\text { treatment naïve subset: } \\
\text { I } 2.7 \text { months vs } 9.1 \text { months, } \\
P=0.037\end{array}$ & 24 \\
\hline Sorafenib & VEGF TKI & Approved & Placebo & $\begin{array}{l}\text { Prior cytokine } \\
\text { allowed }\end{array}$ & PFS & $\begin{array}{l}\text { PFS } 5.5 \text { months (sorafenib) vs } \\
2.2 \text { months (placebo) } \\
P<0.00000 \text { I }\end{array}$ & 17,18 \\
\hline Sunitinib & VEGF TKI & Approved & IFN & Treatment naïve & PFS & $\begin{array}{l}\text { PFS II months (sunitinib) vs } \\
5 \text { months (IFN), } P=0.00 \text { I }\end{array}$ & 13,14 \\
\hline Temsirolimus & mTORi & Approved & $\begin{array}{l}\text { IFN } \\
\text { Temsirolimus/IFN }\end{array}$ & Treatment naïve & OS & $\begin{array}{l}\text { OS I } 0.9 \text { months (temsirolimus) vs } \\
7.3 \text { months (IFN), } P<0.00 \text { I }\end{array}$ & 19 \\
\hline Everolimus & mTORi & Approved & Placebo & $\begin{array}{l}\text { One or two } \\
\text { prior VEGF TKI }\end{array}$ & PFS & $\begin{array}{l}\text { PFS } 4.9 \text { months (everolimus) vs } \\
\text { I.9 months (placebo), } P=0.00 \mathrm{I}\end{array}$ & 20,21 \\
\hline $\begin{array}{l}\text { Bevacizumab/ } \\
\text { IFN }\end{array}$ & $\begin{array}{l}\text { Monoclonal } \\
\text { VEGF Ab }\end{array}$ & Approved & IFN & Treatment naïve & PFS & $\begin{array}{l}\text { AVOREN trial: PFS } 10.2 \text { months } \\
\text { (bevacizumab/IFN) vs } 5.4 \text { months } \\
\text { (IFN), } P<0.000 \text { I } \\
\text { CALGB trial: PFS } 8.5 \text { months } \\
\text { (bevacizumab/IFN) vs } 5.2 \text { months } \\
\text { (IFN), } P=0.000 \text { I }\end{array}$ & $9-12$ \\
\hline Pazopanib & VEGF TKI & Approved & Placebo & $\begin{array}{l}\text { Treatment naive or } \\
\text { prior cytokine } \\
\text { allowed }\end{array}$ & PFS & $\begin{array}{l}\text { PFS } 9.2 \text { months (pazopanib) vs } \\
4.2 \text { months (placebo) } \\
P<0.000 \text { I }\end{array}$ & 15 \\
\hline Axitinib & VEGF TKI & Approved & Sorafenib & $\begin{array}{l}\text { One prior therapy } \\
\text { (sunitinib, } \\
\text { bevacizumab, } \\
\text { cytokine, or } \\
\text { temsirolimus) allowed }\end{array}$ & PFS & $\begin{array}{l}\text { PFS } 6.7 \text { months (axitinib) vs } \\
4.7 \text { months (sorafenib) } \\
P<0.000 \text { I }\end{array}$ & 16 \\
\hline
\end{tabular}

Abbreviations: Ab, antibody; IFN, interferon; mTORi, mammalian target of rapamycin inhibitor; OS, overall survival; PFS, progression-free survival; RCC, renal cell carcinoma; VEGF TKI, vascular endothelial growth factor tyrosine kinase inhibitor; MOA, mechanism of action; FDA, Federal Drug Administration.

and no concomitant anticancer therapy (except for hormonal treatments) or symptomatic central nervous system (CNS) metastases. The drug was to be taken 1 hour prior to meals and for 4 weeks on and 2 weeks off. Patients with

Table 2 Targeted therapies for RCC based on clinical setting and pivotal Phase III trial design

\begin{tabular}{ll}
\hline Clinical setting & Targeted agents \\
\hline Treatment naïve with good or & Tivozanib* \\
intermediate risk MSKCC features & Pazopanib \\
& Sunitinib \\
& Bevacizumab plus interferon \\
Treatment naïe with poor risk & Temsirolimus \\
MSKCC features & Tivozanib* \\
Prior front-line cytokine & Pazopanib \\
& Sorafenib \\
& Axitinib \\
Prior VEGF inhibitor & Everolimus \\
Prior temsirolimus & Axitinib \\
\hline
\end{tabular}

Note: *Tivozanib is not yet FDA approved for RCC in these clinical settings. Abbreviations: RCC, renal cell carcinoma; VEGF, vascular endothelial growth factor; MSKCC, Memorial Sloan Kettering Cancer Center. the following tumor histologies were enrolled: colorectal $(\mathrm{n}=10), \operatorname{RCC}(\mathrm{n}=9)$, pancreatic $(\mathrm{n}=6)$, non-small cell lung cancer $(n=3)$, esophageal $(n=2)$, melanoma $(n=2)$, hepatocellular $(n=2)$, and other $(n=7)$.

Based on animal models, an initial dose of $2.0 \mathrm{mg}$ daily was employed as the starting dose for the trial. However, DLTs were seen at this dose level including hypertension, proteinuria, and ataxia. This led to a subsequent cohort of patients treated at $1.0 \mathrm{mg}$ in which no DLTs were observed, followed by a cohort of $1.5 \mathrm{mg}$ treated patients which was also well tolerated. The $1.5 \mathrm{mg}$ daily dose 4 weeks on, 2 weeks off was determined to be the MTD and the appropriate dose for future studies. At this dose level the most frequent adverse events ( $\geq 25 \%$ ) included hypertension, hoarseness, fatigue, headache, diarrhea, and rash. The mean half-life of the drug was found to be 4.7 days (range 1.3-9.7 days).

From a pharmacodynamic standpoint, measured serum VEGF levels were found to rise with administration of tivozanib while serum VEGF receptor levels were noted to decrease, both in a dose-dependent manner. Additionally, dynamic contrast enhanced magnetic resonance imaging 
was performed in eight patients and showed a trend towards decreased intratumoral vascularity. In terms of activity, $35 \%$ of patients were found to have shrinkage of tumor size, including one RCC patient who had a partial response. Based on the positive findings in this trial, tivozanib was taken into subsequent clinical trials in advanced RCC.

\section{Randomized discontinuation Phase II study of tivozanib in RCC}

Between October 2007 and July 2008, a Phase II randomized discontinuation study of tivozanib in patients with locally advanced or metastatic RCC was performed. ${ }^{24}$ This trial included 272 patients who were either treatment naïve RCC (54\%) or had received only one prior systemic therapy which could not include a VEGF pathway inhibitor. Key exclusion criteria included CNS metastasis, clinically symptomatic metastatic disease, uncontrolled hypertension, recent myocardial infarction (within 3 months of enrollment), and left ventricular dysfunction.

This randomized discontinuation study involved treating patients for 16 weeks followed by assessment of tumor response (Figure 1). Patients who had $\geq 25 \%$ shrinkage were continued on tivozanib and those with $\geq 25 \%$ growth were required to discontinue treatment. Those with responses in between $25 \%$ growth and $25 \%$ shrinkage were blindly randomized $(1: 1)$ to either continuation of tivozanib and placebo. After another 12 weeks of treatment patients were unblinded and restaging scans were performed. Primary endpoints of the trial included safety, objective response rate (ORR, frequency of patients having $\geq 30 \%$ shrinkage) after the first 16 weeks of treatment, and the percent of

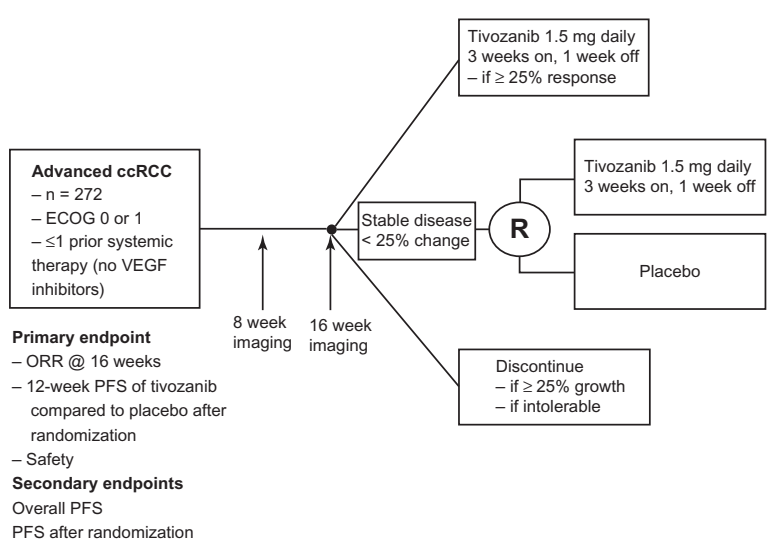

Figure I Schema for Phase II tivozanib trial.

Abbreviations: ccRCC, clear cell renal cell carcinoma; ECOG, Eastern Cooperative Oncology Group (performance score); ORR, objective response rate; PFS, progression-free survival; R, randomized; VEGF, vascular endothelial growth factor. randomized patients who were progression-free after the end of the 12-week continuation of treatment.

Of the patients included in the trial, $83 \%$ had clear cell histology and $73 \%$ had undergone a prior nephrectomy. During the first phase of the study, 78 patients $(29 \%)$ were found to have a response $\geq 25 \%$ at the 16 -week mark, 118 patients (43\%) were eligible for randomization, 50 patients discontinued due to $\geq 25 \%$ growth of disease, and 26 discontinued for other reasons. At the 16-week point, the ORR was $18 \%$ with $66 \%$ having stable disease. In those patients that underwent randomization, the 12-week progression rate was $49 \%$ for those continued on tivozanib compared to the placebo group in which $21 \%$ were progression-free $(P=0.001)$. The median progressionfree survival (PFS) was also significantly prolonged in the tivozanib group (10.3 months for tivozanib compared to 3.3 months for placebo, $P=0.01$ ). Evaluation of ORR for all patients throughout the study showed a rate of $24 \%$ with a total of $84 \%$ of patients having at least some shrinkage in their tumors. The median PFS of all patients treated in the trial was 11.7 months.

Subset analysis of patients with clear cell RCC who had a prior nephrectomy showed an objective response rate of $30 \%$ with a median PFS of 14.8 months. The most common side-effects included hypertension and dysphonia, with less frequent side-effects including diarrhea, proteinuria, fatigue, asthenia, stomatitis, and hand-foot syndrome. Grade 3 or higher adverse events were uncommon and included hypertension (11\%) and elevated serum gammaglutamyl transpeptidase (16\%). Based on the activity and tolerability of tivozanib which was observed in this study, a registrational randomized Phase III trial was undertaken.

\section{Pivotal Phase III study of tivozanib in advanced clear cell RCC}

The Tivozanib Versus Sorafenib in 1st line RCC (TIVO-1) trial was a randomized Phase III trial which compared tivozanib to sorafenib in patients with advanced clear cell RCC. ${ }^{25}$ This trial consisted of 517 patients who were randomized 1:1 to receive either tivozanib at $1.5 \mathrm{mg}$ daily for 3 weeks on and 1 week off or sorafenib $400 \mathrm{mg}$ twice daily continuously (Figure 2). Key eligibility criteria included clear cell histology, prior nephrectomy, ECOG $\leq 1$, adequate organ function, treatment naivety or one prior systemic therapy only (excluding prior VEGF or mTOR inhibitors), and absence of active, untreated CNS metastases. The primary endpoint for the study was PFS with secondary endpoints including safety, response rate, and overall survival. Patients in the 


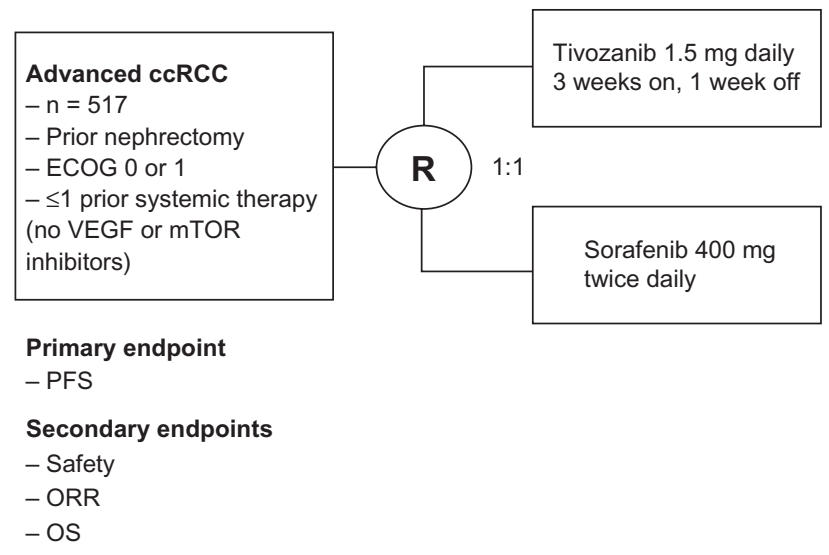

Figure 2 Schema for Phase III tivozanib trial.

Abbreviations: ccRCC, clear cell renal cell carcinoma; ECOG, Eastern Cooperative Oncology Group (performance score); mTOR, mammalian target of rapamycin; ORR, objective response rate; OS, overall survival; PFS, progression-free survival; R, randomization; TIVO-I, Tivozanib Versus Sorafenib in Ist line Renal Cell Carcinoma; VEGF, vascular endothelial growth factor.

sorafenib arm were allowed to cross-over to receive tivozanib after radiographic progression in a separate protocol. Patient characteristics evaluated in the trial showed that $70 \%$ of the patients in both arms were treatment-naïve while $30 \%$ had one prior therapy. The two arms were well balanced in most categories, with slightly more good performance score and MSKCC risk score patients in the sorafenib arm.

The median PFS (independent review) for tivozanib was found to be 11.9 months compared to 9.1 months for sorafenib (hazard ratio [HR] 0.797, $P=0.042$ ). Investigator-reviewed PFS was somewhat better with a median PFS of 14.7 months for tivozanib versus a median PFS of 9.6 months for sorafenib (HR 0.722, $P=0.003$ ). Patients were stratified based on those having prior treatment compared to those who were treatment naïve. Patients in the treatment naïve stratum had a median PFS of 12.7 months compared to 9.1 months for sorafenib $(P=0.037)$. Subgroup analyses showed that patients receiving tivozanib had a benefit over sorafenib when evaluated according to age, sex, ethnicity, ECOG performance score, number of metastatic lesions, and MSKCC risk score (with the exception of poor risk which tended to favor sorafenib). Evaluation of secondary endpoints showed an ORR of $33 \%$ for tivozanib compared to $23 \%$ for sorafenib $(P=0.014)$.

Safety analyses showed hypertension was the most common adverse event for tivozanib treated patients (46\%, compared to $36 \%$ for sorafenib) and $26 \%$ of the patients had grade 3 hypertension or higher. Other common side-effects seen with tivozanib included diarrhea, fatigue, and neutropenia. Patients treated with sorafenib experienced higher rates of diarrhea, hand-foot syndrome, and alopecia ( $2 \%$ vs $21 \%$ ); while patients receiving tivozanib had higher rates of hypertension, dysphonia ( $21 \%$ vs $5 \%)$, and back pain (14\% vs $7 \%)$. Hypertension was shown to be a pharmacodynamic marker for tivozanib benefit. Patients with a diastolic blood pressure (DBP) $>90$ had a median PFS of 18.3 months compared to those with DBP $<90$ having a median PFS of 9.1 months $(P=0.001)$. Likewise, those with a systolic blood pressure (SBP) $>140$ had a median PFS of 16.7 months compared to those with a SBP $<140$ having a median PFS of 9.0 months $(P<0.001)$.

A recent update of the data at European Society for Medical Oncology 2012 meeting ${ }^{26}$ highlighted tolerability of tivozanib with fewer dose-reductions compared to sorafenib (11.6\% vs $42.8 \%, P<0.001)$. Additionally, fewer discontinuations due to toxicity were seen ( $4.2 \%$ vs $5.4 \%)$; however, these were in a similar range. There were also fewer grade 3 or higher events for tivozanib treated patients compared to sorafenib (36.3\% vs 51.0\%). Following radiographic disease progression, $53 \%$ of patients receiving sorafenib crossed over to receive tivozanib; while $17 \%$ of those receiving tivozanib received further treatment. While overall survival data are pending, a press release showed preliminary results of the 1-year survival rate of $77 \%$ for tivozanib compared to $81 \%$ for those receiving sorafenib. ${ }^{32}$ Based on the results of this pivotal trial, tivozanib was planned for FDA approval for use in patients with advanced clear cell RCC. However, on May 2nd, 2013, the FDA declined approval due to the lack of evidence of overall survival benefit. (http://investor. aveooncology.com/phoenix.zhtml? $c=219651 \& p=$ irolnewsArticle \&ID $=1828268$ \&highlight $=$ ).

\section{Other trials evaluating tivozanib in RCC}

There are several other trials evaluating different aspects of tivozanib use for patients with metastatic RCC. These include a combination trial, biomarker study, and patient preference trial. A Phase $\mathrm{Ib}$ combination trial of tivozanib with temsirolimus in patients with metastatic RCC who had received one prior VEGF inhibitor has been performed. ${ }^{27}$ The objectives of this trial were to determine the MTD of the two drugs in combination as well as safety, tolerability, and clinical activity. This study has been reported at the 2011 American Society of Clinical Oncology meeting. Patients in the trial received tivozanib 3 weeks on and 1 week off in combination with weekly temsirolimus. The study design was a $3+3$ dose escalation trial. Twenty-eight patients were accrued to the trial and the MTD was found 
to be the full dose of both agents $(1.5 \mathrm{mg}$ daily for tivozanib and $25 \mathrm{mg}$ weekly for temsirolimus). The adverse events which were commonly seen included fatigue, anorexia, stomatitis, thrombocytopenia, diarrhea, nausea, constipation, and dyspnea. Interestingly, there were no grade 4 or higher events and no DLT. The median duration of treatment was 21.1 weeks (range, 0.3-94.0 weeks). The response rate was found to be $28 \%$ with all partial responses. There were an additional $64 \%$ of patients with stable disease as the best response. This study highlights the tolerability of tivozanib in combination with another agent and makes it a desirable potential backbone of future combination trials. Further updates of this study are awaited.

Another study which has been fully accrued is a biomarker trial exploring candidate biomarkers for tivozanib response. The Biomarker Assessment of Tivozanib in Oncology (BATON) trial included 105 RCC patients who were treated with tivozanib. ${ }^{28}$ Patients were required to have prior nephrectomy, had to receive no more than one systemic therapy (no VEGF or mTOR inhibitors allowed), and be ECOG 0 or 1 . Both clear cell and non-clear cell histologies (up to $30 \%$ of the trial population) were allowed and patients were stratified based on this feature. Patients were treated with open-label tivozanib at a dose of $1.5 \mathrm{mg}$ daily 3 weeks on, 1 week off until disease progression or intolerability. The primary objective of the trial was to correlate the biomarkers from blood and archived tumor samples with clinical activity. Secondary objectives included measurements of ORR, PFS, safety, and correlation of biomarkers with serum concentration of tivozanib. There are several biomarkers being explored in this study including both tumor and blood markers. Tumor biomarkers which will be explored include expression of CD68, HIF1/2, VEGF A-D, hepatocyte growth factor (HGF), carbonic anhydrase 9, and placental growth factor (PLGF). Additionally, a tumor microarray of a 42-gene resistance signature, which defines a possible myeloid resistance mechanism to tivozanib, will be explored. This particular signature was found to correlate with tivozanib resistance in a retrospective analysis in a subset of patients in the Phase II tivozanib trial. ${ }^{29}$ Blood markers which will be explored include VEGF A-D levels, HGF levels, PLGF levels, protein expression, and metabolite patterns. The results of this study are highly anticipated as no current molecular markers are available to help guide therapy in RCC.

Finally, the TivozAnib Use veRsUs Sutent in advanced RCC (TAURUS) study is a recently opened randomized trial comparing patient preference of tivozanib or sunitinib

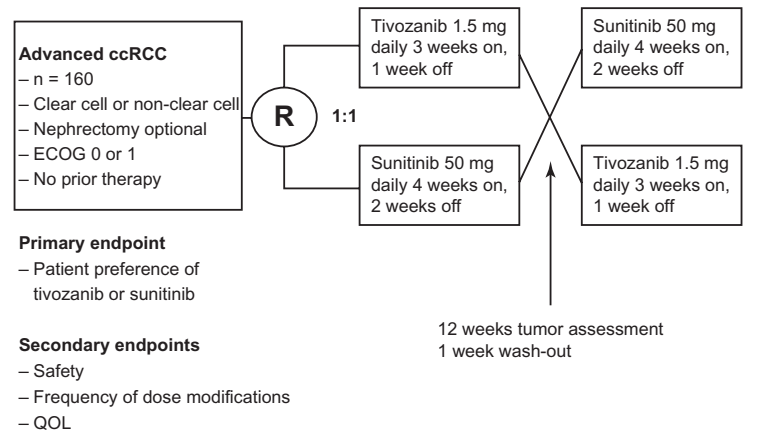

Figure 3 Schema for the TAURUS (TivozAnib Use veRsUs Sutent in advanced renal cell carcinoma) trial.

Abbreviations: ccRCC, clear cell renal cell carcinoma; ECOG, Eastern Cooperative Oncology Group (performance score); QOL, quality of life; R, randomization.

(clinical trial.gov identifier \#NCT01673386). ${ }^{30}$ This is a Phase II, double blind, multinational trial which is planned to enroll 160 patients. The study design (Figure 3) involves randomization $1: 1$ to receive either tivozanib $(1.5 \mathrm{mg}$ daily 3 weeks on, 1 week off) or sunitinib (50 mg daily 4 weeks on, 2 weeks off). Patients will be treated for a 12-week period, followed by tumor assessment and 1-week wash out. Patients will be crossed over to the alternative treatment at this point, despite their response after the first 12 weeks. Patients who are having a response may opt to stay on the same therapy beyond the study (in the case of sunitinib) or in a separate protocol (in the case of tivozanib). Patients who have progressive disease or do not tolerate the treatment in the first period may discontinue the drug early and choose to cross over once their toxicities are improved. The primary endpoint is patient preference of tivozanib or sunitinib. Secondary objectives will be overall safety and tolerability, frequency of dose modifications, and quality of life. Exploratory objectives have been described as antitumor activity at week 12, PFS at 25 weeks, and other quality of life measures and side-effect comparisons. Patients will be stratified based on clear cell and non-clear cell histology as well as ECOG score of 0 or 1 . This study, once resulted, will hopefully give the physician useful information to help with first-line treatment selection, should tivozanib become approved by regulatory agencies.

\section{Discussion}

Tivozanib is a novel orally bioavailable VEGF TKI which has been shown to provide a significant improvement in PFS compared to sorafenib for patients with metastatic clear cell RCC. The drug is yet to receive FDA approval. Based on the information from currently reported trials, should the drug eventually become approved, its position among other agents 
would be as a first line therapy option for patients with clear cell RCC or as an option after a front-line cytokine, such as IL-2. This placement is shown in Table 2, with other firstline VEGF inhibitor treatment options including sunitinib, pazopanib, and bevacizumab plus interferon. For patients with poor risk RCC (as defined by Motzer criteria) or non-clear $\mathrm{RCC}$, temsirolimus remains the agent with a high level of data to support its use in the front-line setting. Unfortunately, the choice of sorafenib as a comparator for the tivozanib frontline trial does not help clinicians choose between tivozanib and other common front-line agents such as sunitinib or pazopanib. In the design stages of TIVO-1, it was likely felt that sorafenib represented the weakest option for a comparator as it is the least potent VEGF TKI receptor inhibitor and had the lowest PFS results in front-line or previously treated RCC patients. However, sorafenib did surprisingly well in the TIVO-1 trial. This could be explained by improved physician experience with using sorafenib over the last several years and their greater comfort with dose reductions and doseinterruptions to help maintain dose intensity.

In clinical practice, sunitinib and pazopanib are commonly used front-line treatment options. These two agents have been compared in a patient preference trial, called PISCES (Patient Preference Study of Pazopanib Versus Sunitinib in Advanced or Metastatic Kidney Cancer). ${ }^{31}$ This study which had a similar design to the ongoing TAURUS study showed that patients preferred pazopanib more commonly compared to sunitinib, with the most common reasons including better quality of life and less fatigue. Although tivozanib has not been compared head-to-head with these agents in the first line setting, there do appear to be differences in terms of side-effect profiles when these agents are compared in their respective Phase III studies. For example, the frequency of fatigue, diarrhea, and hand-foot syndrome for tivozanib was 18\% (Grade 3: 5\%), 22\% (2\%), and 13\% (2\%) compared to sorafenib, which was $16 \%(4 \%), 32 \%(6 \%)$, and $54 \%(17 \%)$, respectively. The frequency of fatigue, diarrhea, and HFS in the sunitinib Phase III trial was 54\% (11\%), 61 (9\%), and 29\% (9\%), respectively. For pazopanib in its Phase III trial, the frequency of these same adverse effects was 55\% (19), 52\% (9\%), and $<10 \%(<1 \%)$, respectively. Given that these sideeffects commonly have a direct impact on quality of life, one would expect that tivozanib would fare well in a head-to-head comparison with a focus on tolerability. This crude cross-trial comparison may help physicians in their selection of several agents in the first line setting as comparative effectiveness data are lacking. In addition to effectiveness and tolerability, the findings from the Biomarker Assessment of Tivozanib in
ONcology (BATON) study may also help physicians in their selection should the infiltrative myeloid cell resistance gene panel turn out to help predict those patients who would be resistant to the agent.

Lastly, there are several points from the TIVO-1 trial which could help give us some perspective on survival data which are becoming available from this study. With numerous active agents available for RCC, it is difficult to measure overall survival differences between agents and therefore, PFS is a necessary end-point for Phase III trials. The overall survival data from the TIVO-1 trial are yet to be reported, although the 1-year survival data has been reported in a press release. ${ }^{32}$ The 1 -year survival for the tivozanib cohort was $77 \%$ and for sorafenib was $81 \%$. This lack of survival difference should not be too surprising given similar studies in this disease which have failed to show statistical differences in overall survival due to availability of other agents. However, other trials do show favorable survival trends with hazard ratios which are $<1$, which is a key difference. Also, there are several clues that hint that this might be the case. First, there is an imbalance of good risk patients and good performance status patients in the sorafenib arm compared to the tivozanib arm. There were 54\% with an ECOG score of 0 in the sorafenib arm compared to $45 \%$ in the tivozanib arm. Additionally, in terms of MSKCC score, there were $24 \%$ good risk, $67 \%$ intermediate risk, and $7 \%$ poor risk patients in the tivozanib arm compared to $34 \%, 62 \%$, and $4 \%$ patients in the sorafenib arm, respectively. These findings could result in a better survival rate in the sorafenib arm as these are known factors which result in better prognosis independent of treatment. Most importantly, there were more patients in the sorafenib arm who were exposed to later effective therapies after progression than in the tivozanib arm. Because of the separate cross-over trial which was offered to those patients treated with sorafenib, 53\% of patients went on to receive tivozanib, while only a small number of patients $(17 \%)$ had subsequent therapy after receiving firstline tivozanib. The low number of patients who received subsequent therapy after tivozanib is likely explained by the large number of patients $(90 \%)$ who were treated in Eastern Europe, where there are few treatment options available for patients with metastatic RCC.

At the crux of this issue is whether PFS remains a valuable primary endpoint for registrational trials when it is unclear if PFS improvements equate to survival improvements. Although PFS may not be an ideal endpoint for clinical studies and may not directly predict overall survival benefits in a disease with numerous effective treatments, certainly it is 
a meaningful palliative endpoint for patients as PFS measures time in which the patient is not having progression of cancer and thus cancer-related symptoms. Until better endpoints are found which accurately describe an agent's effectiveness and ability to improve overall-survival, PFS will remain a commonly used primary measurement. Unfortunately, it seems that we are reaching a ceiling with VEGF TKI agents in RCC therapeutic development and novel agents which have different mechanisms of action are greatly needed.

\section{Conclusion}

Tivozanib is an orally bioavailable VEGF TKI which has a long half-life and excellent potency and specificity to the VEGF receptors. The drug has shown tolerability and efficacy in early phase trials and has shown superiority to sorafenib in terms of improved PFS and acceptable toxicity profile in patients with metastatic clear cell RCC. Key questions which remain include how to select this agent as a front-line option compared to other agents and what is its clinical activity in patients with non-clear cell histologies. There are ongoing trials which will hopefully shed further light on these questions, including the patient-preference TAURUS study and biomarker BATON trial. Given that the FDA has not approved tivozanib based on the results of the TIVO-1 trial, the fate of tivozanib in RCC is currently unclear.

\section{Disclosure}

The author reports no conflicts of interest in this work.

\section{References}

1. Siegel R, DeSantis C, Virgo K, et al. Cancer treatment and survivorship statistics, 2012. CA Cancer J Clin. 2012;62(4):220-241.

2. Kim WY, Kaelin WG. Role of VHL gene mutation in human cancer. J Clin Oncol. 2004;22(24):4991-5004.

3. Wiesener MS, Münchenhagen PM, Berger I, et al. Constitutive activation of hypoxia-inducible genes related to overexpression of hypoxia-inducible factor-1 alpha in clear cell renal carcinomas. Cancer Res. 2001;61(13):5215-5222.

4. Gnarra JR, Tory K, Weng Y, et al. Mutations of the VHL tumour suppressor gene in renal carcinoma. Nat Genet. 1994;7(1):85-90.

5. Shuin T, Kondo K, Torigoe S, et al. Frequent somatic mutations and loss of heterozygosity of the von Hippel-Lindau tumor suppressor gene in primary human renal cell carcinomas. Cancer Res. 1994;54(11): 2852-2855.

6. Carmeliet P. VEGF as a key mediator of angiogenesis in cancer. Oncology. 2005;69 Suppl 3:4-10.

7. Motzer RJ, Russo P. Systemic therapy for renal cell carcinoma. J Urol. 2000;163(2):408-417.

8. Bukowski RM. Cytokine therapy for metastatic renal cell carcinoma. Semin Urol Oncol. 2001;19(2):148-154.

9. Motzer RJ, Mazumdar M, Bacik J, Berg W, Amsterdam A, Ferrara J. Survival and prognostic stratification of 670 patients with advanced renal cell carcinoma. J Clin Oncol. Aug 1999;17(8):2530-2540.

10. Rini BI, Halabi S, Rosenberg JE, et al. Bevacizumab plus interferon alfa compared with interferon alfa monotherapy in patients with metastatic renal cell carcinoma: CALGB 90206. J Clin Oncol. 2008;26(33): $5422-5428$.
11. Rini BI, Halabi S, Rosenberg JE, et al. Phase III trial of bevacizumab plus interferon alfa versus interferon alfa monotherapy in patients with metastatic renal cell carcinoma: final results of CALGB 90206. J Clin Oncol. 2010;28(13):2137-2143.

12. Escudier B, Pluzanska A, Koralewski P, et al; AVOREN Trial investigators. Bevacizumab plus interferon alfa- 2 a for treatment of metastatic renal cell carcinoma: a randomised, double-blind phase III trial. Lancet. 2007;370(9605):2103-2111.

13. Escudier B, Bellmunt J, Négrier S, et al. Phase III trial of bevacizumab plus interferon alfa-2a in patients with metastatic renal cell carcinoma (AVOREN): final analysis of overall survival. J Clin Oncol. 2010; 28(13):2144-2150.

14. Motzer RJ, Hutson TE, Tomczak P, et al. Sunitinib versus interferon alfa in metastatic renal-cell carcinoma. $N$ Engl J Med. 2007;356(2): 115-124.

15. Motzer RJ, Hutson TE, Tomczak P, et al. Overall survival and updated results for sunitinib compared with interferon alfa in patients with metastatic renal cell carcinoma. J Clin Oncol. 2009;27(22): 3584-3590.

16. Sternberg CN, Davis ID, Mardiak J, et al. Pazopanib in locally advanced or metastatic renal cell carcinoma: results of a randomized phase III trial. J Clin Oncol. 2010;28(6):1061-1068.

17. Rini BI, Escudier B, Tomczak P, et al. Comparative effectiveness of axitinib versus sorafenib in advanced renal cell carcinoma (AXIS): a randomised phase 3 trial. Lancet. 2011;378(9807): 1931-1939.

18. Escudier B, Eisen T, Stadler WM, et al. Sorafenib in advanced clear-cell renal-cell carcinoma. $N$ Engl J Med. 2007;356(2):125-134.

19. Escudier B, Eisen T, Stadler WM, et al. Sorafenib for treatment of renal cell carcinoma: Final efficacy and safety results of the phase III treatment approaches in renal cancer global evaluation trial. $J$ Clin Oncol. 2009;27(20):3312-3318.

20. Hudes G, Carducci M, Tomczak P, et al; Global ARCC Trial. Temsirolimus, interferon alfa, or both for advanced renal-cell carcinoma. N Engl J Med. 2007;356(22):2271-2281.

21. Motzer RJ, Escudier B, Oudard S, et al; RECORD-1 Study Group. Efficacy of everolimus in advanced renal cell carcinoma: a double-blind, randomised, placebo-controlled phase III trial. Lancet. 2008;372(9637):449-456.

22. Motzer RJ, Escudier B, Oudard S, et al; RECORD-1 Study Group. Phase 3 trial of everolimus for metastatic renal cell carcinoma: final results and analysis of prognostic factors. Cancer. 2010;116(18): 4256-4265.

23. Eskens FA, de Jonge MJ, Bhargava P, et al. Biologic and clinical activity of tivozanib (AV-951, KRN-951), a selective inhibitor of VEGF receptor-1, -2, and -3 tyrosine kinases, in a 4-week-on, 2-week-off schedule in patients with advanced solid tumors. Clin Cancer Res. 2011;17(22):7156-7163.

24. Nosov DA, Esteves B, Lipatov ON, et al. Antitumor activity and safety of tivozanib (AV-951) in a phase II randomized discontinuation trial in patients with renal cell carcinoma. J Clin Oncol. 2012; 30(14):1678-1685.

25. Motzer RJ, Nosov D, Eisen T, et al. Tivozanib versus sorafenib as initial targeted therapy for patients with advanced renal cell carcinoma: Results from a phase III randomized, open-label, multicenter trial. J Clin Oncol. 2012;30 Suppl:Abstr 4501.

26. Eisen T, Sternberg CN, et al. Detailed comparison of the safety of tivozanib hydrochloride versus sorafenib in patients with advanced/ metastatic renal cell carcinoma (mRCC) from a Phase III trial. European Society of Medical Oncology 2012 Congress. Abstract 795PD.

27. Kabbinavar FF, Srinivas S, Hauke RJ, et al. Results from a phase I trial of tivozanib (AV-951) combined with temsirolimus therapy in patients (pts) with renal cell carcinoma (RCC). J Clin Oncol. 2011;29 Suppl: Abstr 4549.

28. Hutson TE, Rathmell K, Hudes GR, et al. A phase II biomarker assessment of tivozanib in oncology (BATON) trial in patients (pts) with advanced renal cell carcinoma (RCC). J Clin Oncol. 2012; 30 Suppl:Abstr TPS4686. 
29. Robinson MO, Lin J, Feng B, et al. Correlation of a tivozanib response biomarker identified in a preclinical model with clinical activity in a phase II study in renal cell carcinoma (RCC). J Clin Oncol. 2010; 28 Suppl:Abstr e13564.

30. Pfizer. A study in patients with non-small cell lung cancer testing if erlotinib plus SU011248 (sunitinib) is better than erlotinib alone (SUN1058). In: ClinicalTrials.gov [website on the Internet]. Bethesda, MD: US National Library of Medicine; 2011 [updated August 26, 2011]. Available from: http://www.clinicaltrials.gov/ct2/show/NCT00265317. NLM identifier: NCT00265317. Accessed August 1, 2011.
31. Escudier BJ, Porta C, Bono P, et al. Patient preference between pazopanib (Paz) and sunitinib (Sun): Results of a randomized double-blind, placebo-controlled, cross-over study in patients with metastatic renal cell carcinoma (mRCC) - PISCES study, NCT 01064310. J Clin Oncol. 2012;30 Suppl:Abstr CRA4502.

32. AVEO Oncology. AVEO Reports Second Quarter 2012 Financial Results [press release]. Cambridge, MA: AVEO Oncology; 2012. Available from: http://investor.aveooncology.com/phoenix.zhtml?c=219651\&p=irolnewsArticle \&ID=1721417\&highlight $=$. Accessed November 15, 2012.

\section{Publish your work in this journal}

Drug Design, Development and Therapy is an international, peerreviewed open-access journal that spans the spectrum of drug design and development through to clinical applications. Clinical outcomes, patient safety, and programs for the development and effective, safe, and sustained use of medicines are a feature of the journal, which has also been accepted for indexing on PubMed Central. The manuscript management system is completely online and includes a very quick and fair peer-review system, which is all easy to use. Visit http://www.dovepress.com/testimonials.php to read real quotes from published authors.

Submit your manuscript here: http://www.dovepress.com/drug-design-development-and-therapy-journal 